\title{
Photodissociation of the $\mathrm{HeH}^{+}$molecular ion
}

\author{
Irina Dumitriu and Alejandro Saenz \\ Humboldt-Universität zu Berlin, Institut für Physik, AG Moderne Optik, \\ Hausvogteiplatz 5-7, D-10117 Berlin, Germany. \\ E-mail: Alejandro.Saenz@physik.hu-berlin.de
}

\begin{abstract}
The photodissociation cross section of the molecular ion $\mathrm{HeH}^{+}$was calculated within the Born-Oppenheimer approximation for a parallel, a perpendicular, and an isotropic orientation of the molecular axis with respect to the field, considering also different initial vibrational and rotational states. The results were compared to recent data from a free-electron laser experiment performed at the FLASH facility [H.B. Pedersen et al., Phys. Rev. Lett.98, 223202, (2007)]. Within the experimental uncertainties theoretical and experimental results are compatible with each other.
\end{abstract}

PACS numbers: 31.50.Gh, 33.80.Gj

Submitted to: J. Phys. B: At. Mol. Phys. 


\section{Introduction}

The helium-hydride ion $\mathrm{HeH}^{+}$is the simplest heteronuclear two-electron system and the only well bound molecular system of hydrogen and helium, the most abundant elements in the universe. This motivated many calculations and experiments performed on this molecular ion which is of interest for the chemistry of astrophysical objects, for the tritium neutrino mass experiments, or simply by itself as a model system (see, e.g., [1, 2, 3, 4, 5, 6, 7, 8, 9, 10, and references therein). More recently, $\mathrm{HeH}^{+}$is drawing special attention due to an experiment performed at the free-electron laser FLASH [11] where the absolute photodissociation cross section at a photon energy of $38.7 \mathrm{eV}$ was measured.

The photoabsorption spectrum of $\mathrm{HeH}^{+}$was previously investigated theoretically, together with photoionization, only for the parallel orientation of the molecular axis with respect to the field [8]. A more recent study on the photoionization of $\mathrm{HeH}^{+}[12$ treated also the perpendicular orientation, but for the photodissociation into electronic bound states, to the authors' knowledge, the only work considering both the parallel and the perpendicular orientation is still the one of Basu and Barua from 1984 [7]. There, however, the $\Pi$ contribution taken into account is only the one of the lowest state, while the FLASH experiment shows an important contribution of higher lying $\Pi$ states [11.

Motivated by the FLASH experiment, the photodissociation cross section of $\mathrm{HeH}^{+}$ has been calculated for parallel, perpendicular, as well as isotropic orientations of the molecular axis with respect to the field, for transitions starting from the lowest lying vibrational state and the first or second rotational level, as well as for a mixture of initial vibrational states. Since the experiment measures only the dissociation into $\mathrm{He}+\mathrm{H}^{+}$, a comparison to the experimental data required resolving different dissociation channels. The influence of non-adiabatic effects on the channel-resolved results is discussed. The paper is organized as follows: section 2 presents the method and the computational details, section 3 discusses the results and a summary is given in Section 4.

\section{Method and computational details}

In atomic units $\left(1 E_{h}=27.21139 \mathrm{eV}\right.$ and $\left.1 a_{0}=5.29177 \cdot 10^{-11} \mathrm{~m}\right)$ and the nonrelativistic dipole approximation the photodissociation cross section is given according to Fermi's Golden Rule by

$$
\sigma(\omega)=g \frac{4 \pi^{2}}{c} \omega\left|\left\langle\Psi_{n^{\prime} \varepsilon^{\prime} J^{\prime} m_{J}^{\prime}}|\hat{D}| \Psi_{n \nu J m_{J}}\right\rangle\right|^{2} \cdot \delta\left(\omega-\left[E_{n^{\prime} \varepsilon^{\prime} J^{\prime} m_{J}^{\prime}}-E_{n \nu J m_{J}}\right]\right)
$$

where $\omega$ is the photon energy and $\hat{D}$ is the dipole operator in length gauge. For a diatomic molecule, it is sufficient to calculate the single-photon absorption cross section for a parallel and a perpendicular orientation of the molecular axis with respect to the electric field component. The partial parallel (perpendicular) cross section for an isotropic molecular ensemble is then obtained by a multiplication of the cross section for a molecule oriented parallel (perpendicular) to the field with the statistical factor 
$g=1 / 3(g=2 / 3)$. The total cross section for an isotropic molecular ensemble is simply the sum of these two partial cross sections (see also [8, 13] for more details on $g$ ).

In equation (11), $\Psi_{n \nu J m_{J}}$ and $\Psi_{n^{\prime} \varepsilon^{\prime} J^{\prime} m_{J}^{\prime}}$ are the rovibronic wavefunctions of the initial and final state, with energies $E_{n \nu J m_{J}}$ and $E_{n^{\prime} \varepsilon^{\prime} J^{\prime} m_{J}^{\prime}}$ respectively. Within the BornOppenheimer (BO) approximation the former is given as

$$
\Psi_{n \nu J m_{J}}\left(\vec{r}_{1}, \vec{r}_{2}, \vec{R}\right)=\psi_{n}\left(\vec{r}_{1}, \vec{r}_{2} ; R\right) \frac{\Phi_{\nu J}^{(n)}(R)}{R} Y_{J m_{J}}(\Omega)
$$

where $\psi_{n}\left(\vec{r}_{1}, \vec{r}_{2} ; R\right)$ is the electronic, $\Phi_{\nu J}^{(n)}(R) / R$ the vibrational, and the spherical harmonic $Y_{J m_{J}}$ the rotational wavefunction. The index $n$ specifies the electronic state (BO potential curve) including its symmetry (the only relevant ones in this work are ${ }^{1} \Sigma$ and ${ }^{1} \Pi$ ), while $\nu$ and $\left\{J, m_{J}\right\}$ are the vibrational and rotational quantum numbers, respectively. The final-state wavefunction can be written in an analogous way, but in the here studied case of photodissociation $\varepsilon^{\prime}$ is a continuous index, while $n, n^{\prime}, \nu$, $J$, and $J^{\prime}$ are discrete ones. Correspondingly, $\psi_{n}, \psi_{n^{\prime}}, \Phi_{\nu J}^{(n)}$, and, of course, $Y_{J m_{J}}$ as well as $Y_{J^{\prime} m_{J}^{\prime}}$ are normalized to unity, while $\Phi_{\varepsilon^{\prime} J^{\prime}}^{\left(n^{\prime}\right)}$ is energy normalized, $\left\langle\Phi_{\varepsilon^{\prime} J^{\prime}}^{\left(n^{\prime}\right)} \mid \Phi_{\varepsilon^{\prime \prime} J^{\prime}}^{\left(n^{\prime}\right)}\right\rangle=\delta\left(\varepsilon^{\prime}-\varepsilon^{\prime \prime}\right)$.

The transition amplitudes in equation (11) are obtained by a calculation of the integral

$$
\left\langle\Psi_{n^{\prime} \varepsilon^{\prime} J^{\prime} m_{J}^{\prime}}|\hat{\mathrm{D}}| \Psi_{n \nu J m_{J}}\right\rangle=\int \Phi_{\varepsilon^{\prime} J^{\prime}}^{*\left(n^{\prime}\right)}(R) D_{n^{\prime} n}(R) \Phi_{\nu J}^{(n)}(R) \mathrm{d} R
$$

where $D_{n^{\prime} n}(R)$ denotes the electronic transition dipole moment as a function of the internuclear distance. It is defined by

$$
D_{n^{\prime} n}(R)=\int \psi_{n^{\prime}}^{*}\left(\vec{r}_{1}, \vec{r}_{2} ; R\right) \hat{d} \psi_{n}\left(\vec{r}_{1}, \vec{r}_{2} ; R\right) \mathrm{d}^{3} r
$$

where $\hat{d}$ is the electronic dipole operator in length gauge, $\hat{d}=\vec{\epsilon} \cdot\left(\vec{r}_{1}+\vec{r}_{2}\right)$ with $\vec{\epsilon}$ being the polarization vector. (The contribution of the nuclear dipole operator vanishes, since in the present work only electronic transitions with $n \neq n^{\prime}$ are considered.)

The electronic wavefunctions and transition dipole moments are calculated using the method described in [14] which is thus only briefly sketched in the following. First, the one-electron Schrödinger equation (OESE) is solved in an elliptical "box" within the fixed-nuclei approximation, using a $B$-spline basis set and the prolate-spheroidal coordinates $(1 \leq \xi<\infty,-1 \leq \eta \leq 1,0 \leq \phi<2 \pi)$. The box boundary is defined by $\xi_{\max }$. The two-electron Schrödinger equation is solved using the configurationinteraction (CI) method in which the configurations are expanded in terms of the oneelectron orbitals obtained from the solution of the OESE. This yields the BO potential curves and the two-electron wavefunctions $\psi$. The latter are used to evaluate the electronic transition dipole moments $D_{n^{\prime} n}$. All the results shown in this work were calculated in the length gauge, but agreement with velocity gauge was also investigated.

The nuclear motion is solved in the adiabatic BO potential curves by expanding the nuclear wavefunctions in $B$ splines for the radial part times spherical harmonics describing the angular part. The radial Schrödinger equation is solved in a spherical "box" whose boundary is defined by $R_{\max }$. The box leads to a discretization of the 
vibrational continua. The resulting vibrational wavefunctions $\Phi_{\varepsilon^{\prime} J}^{\left(n^{\prime}\right)}$ are therefore all discrete and normalized to unity. For the dissociative continuum the renormalization factors that lead to an energy normalization can simply be obtained from the density of states.

The present calculations were directly motivated by the recent FEL experiment [1]. Therefore, the basis sets were chosen for the purpose of computing photodissociation cross sections that take into account the contribution of as many Rydberg states as possible (the experimental results indicate the importance of highly excited states). In the present calculation $35 \Sigma$ and $25 \Pi$ electronic bound states were finally included. A physical box $\left(r_{\max }=\frac{R}{2} \xi_{\max }\right)$ equal to $40 a_{0}$ was used, changing the size of the elliptical box $\xi_{\max }$ synchronously with the variation of the internuclear distance $R, 40 B$ splines of order 8 were used for the $\xi$ direction and $20 B$ splines of order 6 for the $\eta$ direction. The BO potential curves were calculated in an $R$ interval in between 0.1 and $60 a_{0}$. The latter limit was mainly needed for a determination of the dissociation channel, i. e. to find out into which atomic or ionic fragments $\mathrm{HeH}^{+}$dissociates. The basis set used for calculating the vibrational wavefunctions $\Phi$ comprised a box $\mathrm{R}_{\max }=20 a_{0}$ and $900 B$ splines of order 10 .

\section{Results}

Calculations were performed for transitions starting from the lowest lying vibrational state of the electronic ground state to all the calculated electronic bound states, for both parallel and perpendicular orientation of the molecular axis with respect to the field. The results shown here are for transitions starting in the rotational level $J=1$, which is the most probable value of $J$ at room temperature [7], and going to $J^{\prime}=0,2$ for the parallel orientation (according to the selection rule $\Delta J= \pm 1, \Delta J \neq 0$ ) and to $J^{\prime}=0,1,2$ for the perpendicular transitions (selection rule: $\Delta \mathrm{J}=0, \pm 1$ ). Since previous calculations [8] were performed for transitions starting from $J=0$, the spectrum for these transitions is also shown for comparison, but the final result is not significantly influenced by the rotational motion within the considered $J$ values.

\subsection{Energies, cross section, and comparison to previous results}

Calculations of electronic energies and dipole moments for different values of the internuclear separation were performed. For checking the accuracy of these calculations, results were compared to literature $([2,3,8])$ and good agreement was found for the basis sets described in Section 2. Plots of the computed potential curves for the ${ }^{1} \Sigma$ and ${ }^{1} \Pi$ symmetries are shown in figures 1 and 2, respectively. These potential curves were used in the subsequent calculation of the photodissociation cross sections. Clearly, all potential curves are purely dissociative within the Franck-Condon window of the electronic ground state. One-photon transitions from the ground to the excited states lead therefore to dissociation. 


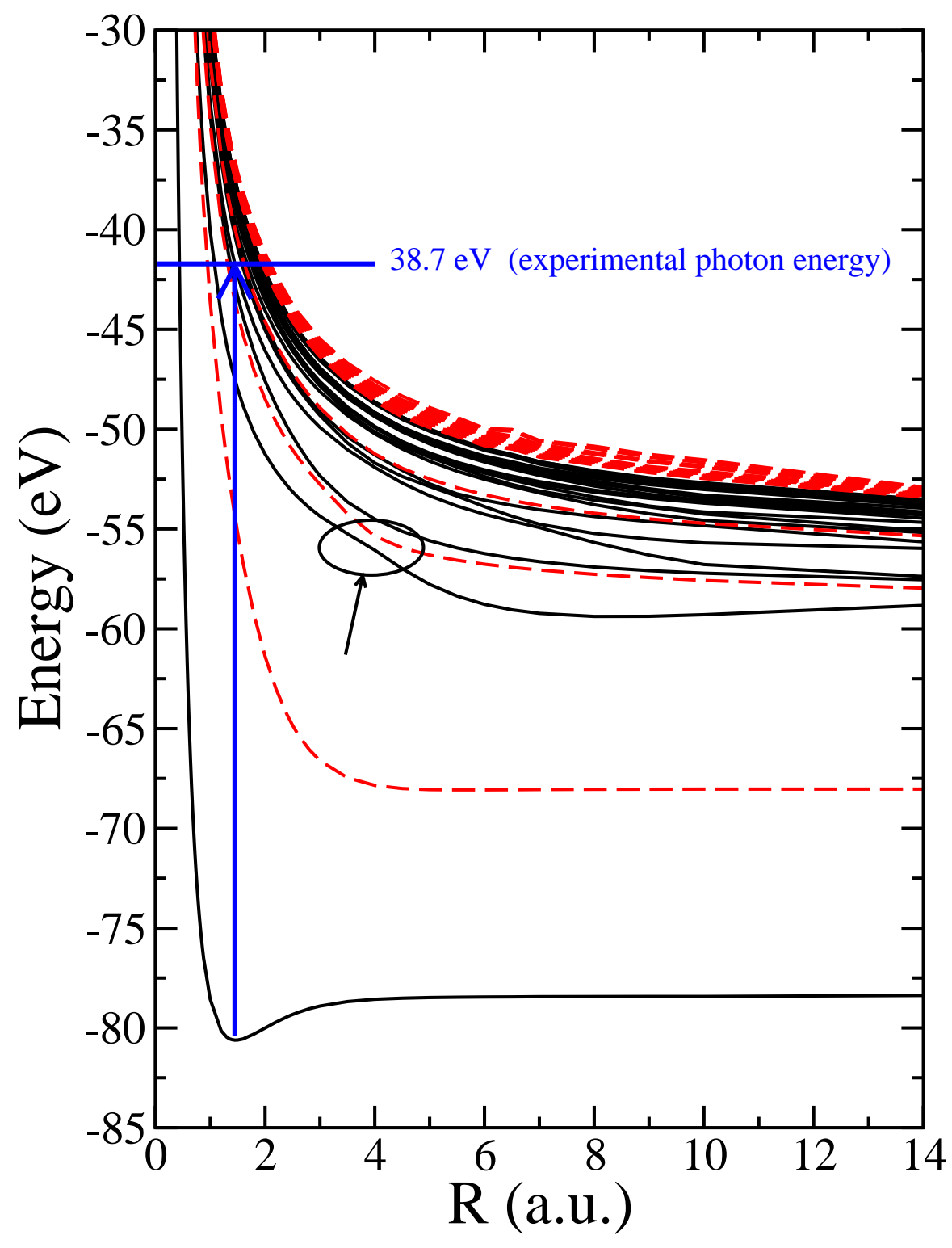

Figure 1. The potential curves of the ${ }^{1} \Sigma$ states of $\mathrm{HeH}^{+}$(included in the present calculation): the full black lines represent states dissociating adiabatically into $\mathrm{He}+$ $\mathrm{H}^{+}$, while the red dashes represent the $\mathrm{H}+\mathrm{He}^{+}$channel. The black arrow points at an (also encircled) avoided crossing that is expected to influence the channel-resolved results. The blue vertical arrow marks the experimental photon energy used in [11.

The gauge dependence of the results was investigated by comparing the electronic transition dipole moments obtained in either the length or the velocity gauge. The latter moments are obtained from equation (4), if the dipole operator is expressed as $\hat{d}_{v}=\vec{\epsilon} \cdot\left(\vec{\nabla}_{1}+\vec{\nabla}_{2}\right)$ and multiplied by $\frac{1}{\omega}$ ( $\omega$ being the photon energy) for comparing to the length gauge results. Figure 3 shows a comparison of the transition dipole moments obtained in the two gauges for the equilibrium internuclear separation $R_{\text {eq }}=1.45 a_{0}$ and for a parallel orientation (transitions to ${ }^{1} \Sigma$ states). The overall agreement is evidently very good. Noticeable deviations are only found for some very high lying electronic 


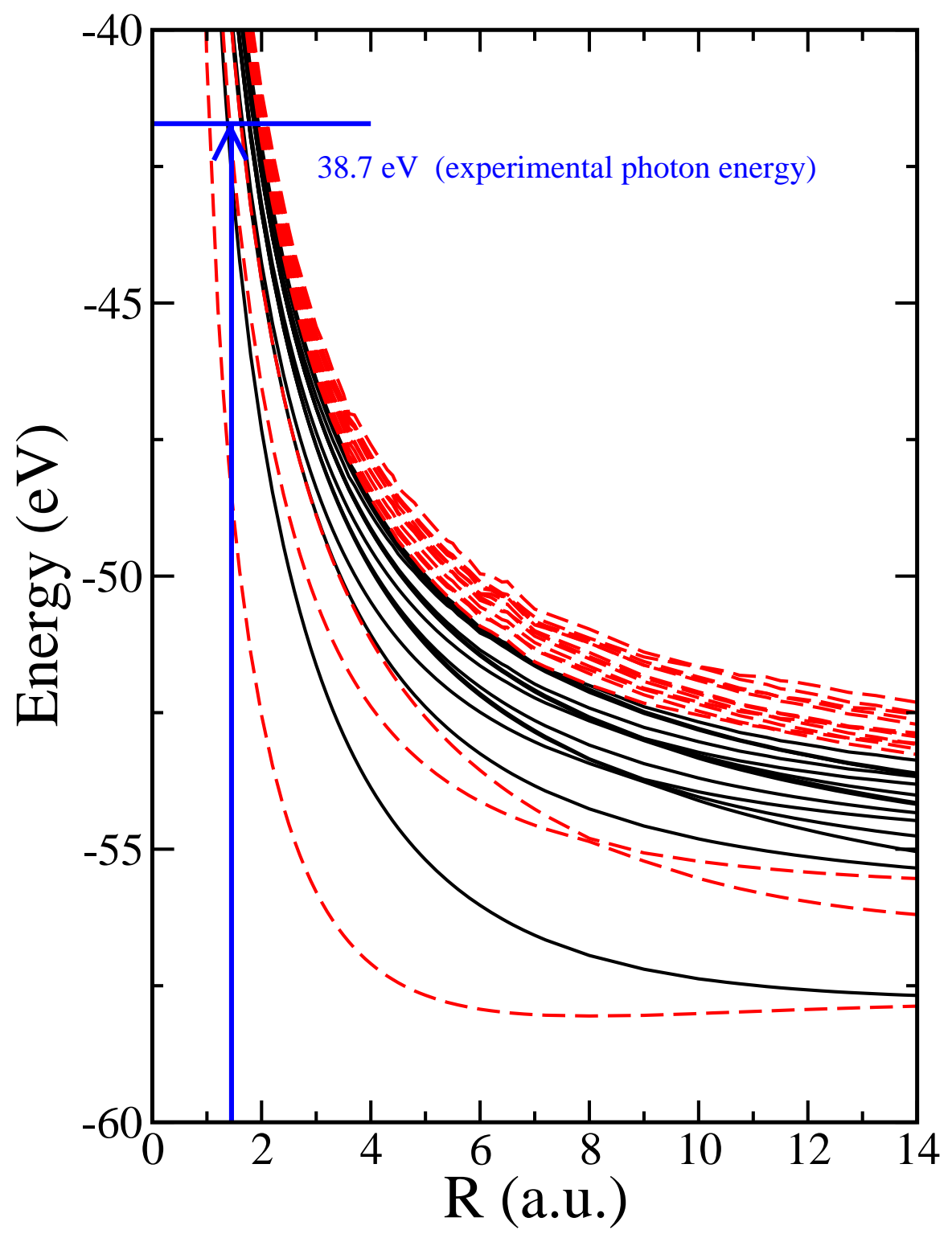

Figure 2. As figure 1, but the ${ }^{1} \Pi$ states are shown.

Rydberg states that possess, however, a very small transition moment. These deviations do therefore have an extremely small influence on the cross-sections obtained from all electronic states.

In figure 4 the calculated photodissociation cross section for a parallel alignment of the molecular axis with respect to the photon polarization vector $(g=1)$ is compared to a previous result [8] in which explicitly correlated basis functions had been used for the evaluation of the electronic wavefunctions. Both results were obtained for transitions starting from the absolute rovibronic ground state of the $\mathrm{HeH}^{+}$molecule $(\nu=0, J=0)$. The most evident difference between the two spectra lies in the position and height of their maxima. This difference is due to the fact that the electronic wavefunctions especially for the ground and the first excited states are more accurately 


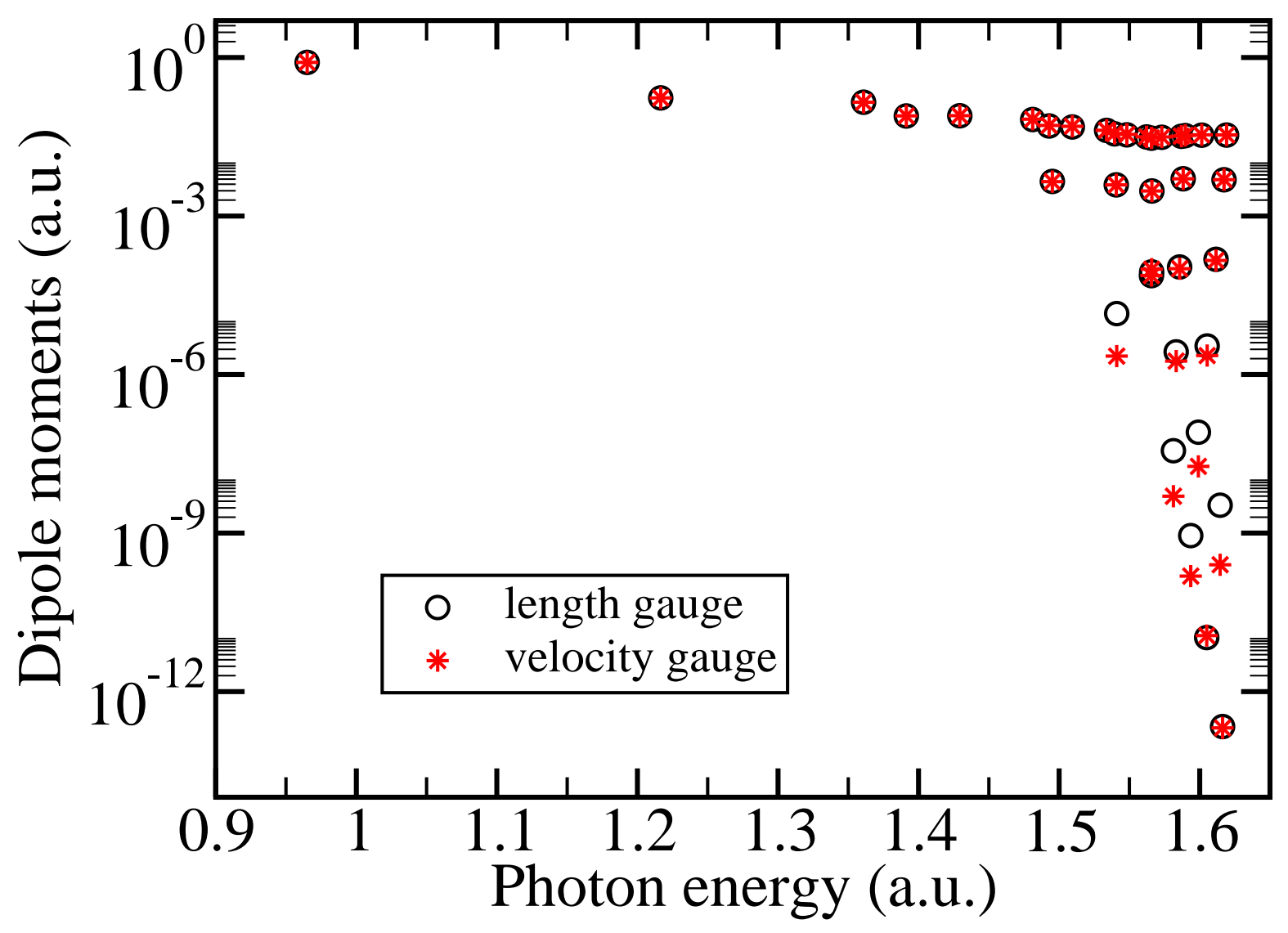

Figure 3. The absolute value of electronic transition dipole moments for the equilibrium internuclear distance $R=1.45 a_{0}$, parallel orientation. The black circles are the length gauge results, while the red crosses are the velocity gauge dipole moments.

represented in [8] than in the present calculation, as is concluded from a comparison of the corresponding energies.

In references [14, 15] it was demonstrated that the present approach can yield extremely accurate two-electron wavefunctions, but this requires a judicious choice of the basis set, if the size of the calculation should be kept reasonably small. Most importantly, different electronic states possess different optimal basis sets. On the other hand, the goal of the present work was to consider as many excited bound states as possible and to calculate all of them with sufficient and comparable accuracy. The most convenient way to do this is to use a single basis set for all states, since it avoids non-orthogonality problems and preserves sum-rule completeness. This leads to a compromise for the results for the ground and first excited states. It was checked that by improving the ground and first excited states the agreement to [8] in the peak region is also improving. The remaining part of the spectrum that stems from transitions to higher-lying electronic states is, however, in good agreement with the previous result in [8]. As is discussed below, for a comparison to the experiment reported in [11] the accuracy of the first excited electronic state plays practically no role and thus the good agreement with the 


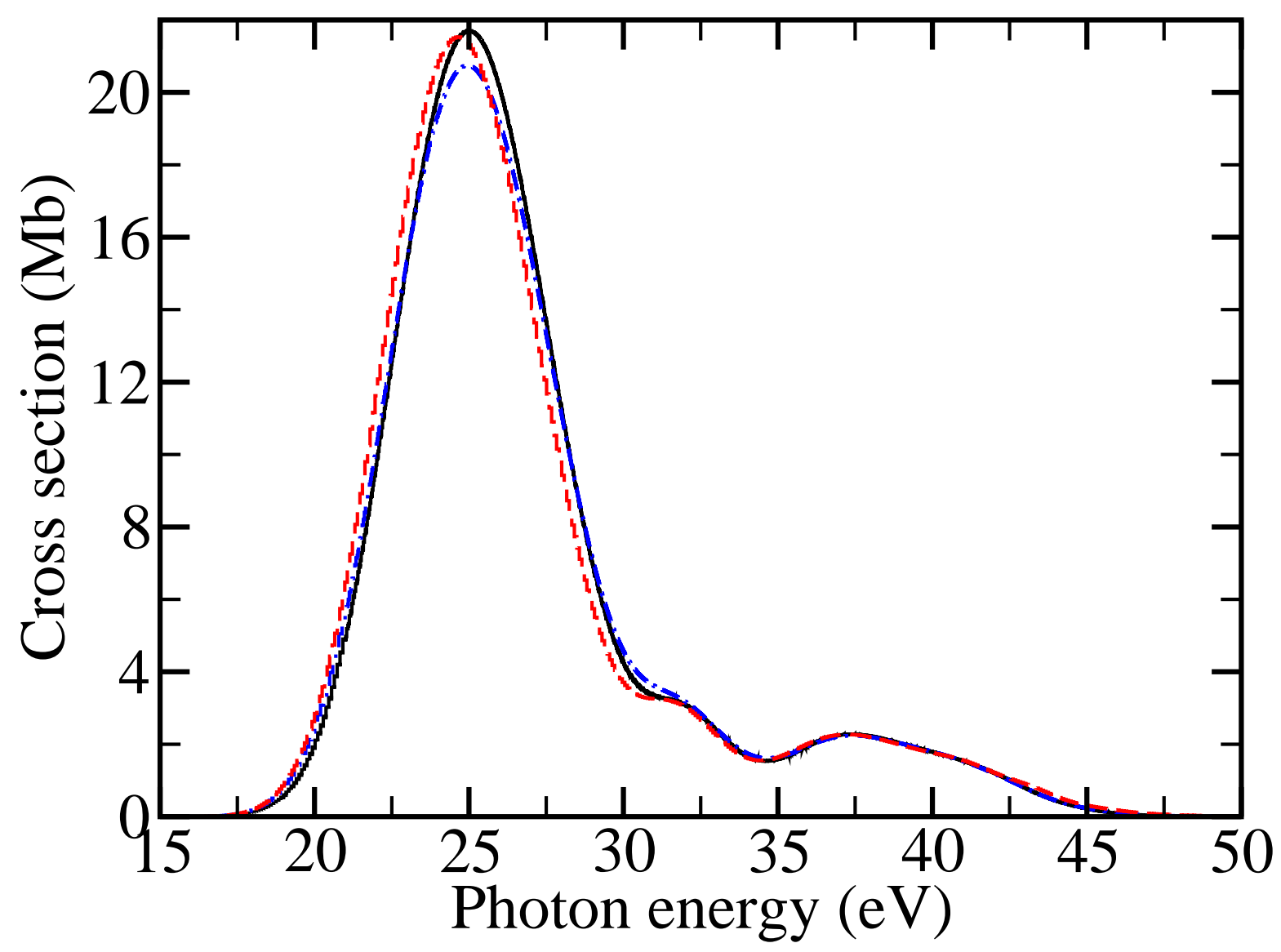

Figure 4. Comparison of the results obtained for the parallel orientation of the molecular axis with respect to the field to the results from [8]: the full black line is the result of the present calculation for $J=1 \rightarrow J^{\prime}=0,2$, the blue chain is present calculation for $J=0 \rightarrow J^{\prime}=1$, and the red dashed line is the result from [8] (obtained for $J=0 \rightarrow J^{\prime}=1$ ).

previous calculation for the higher photon energies is more important for the purpose of this work.

Figure 4 shows also the parallel photodissociation cross section for the first excited rotational state $(J=1)$ which, according to [7], is the most probable rotational level at room temperature. Accidentally, the result for $J=1$ agrees better to the previous theoretical calculation performed for $J=0$. From this accidental agreement and the discussion above it is clear that the influence of rotational excitation is in general rather small and most prominent for the transition to the first excited electronic state. It was, however, checked that the results shown in the following are all relatively insensitive to the initial rotational state, as long as $J$ is not very large.

Figure 5 shows the photodissociation cross section of $\mathrm{HeH}^{+}$for an isotropic orientation of the molecular axis with respect to the field, as well as the parallel $(g=1 / 3)$ and perpendicular $(g=2 / 3)$ contributions to it, for transitions starting from the lowest lying vibrational state $\nu=0$ and the first excited rotational level $J=1$. It can be seen in figure 5 that until about $27.5 \mathrm{eV}$ the spectrum is practically identical to the 


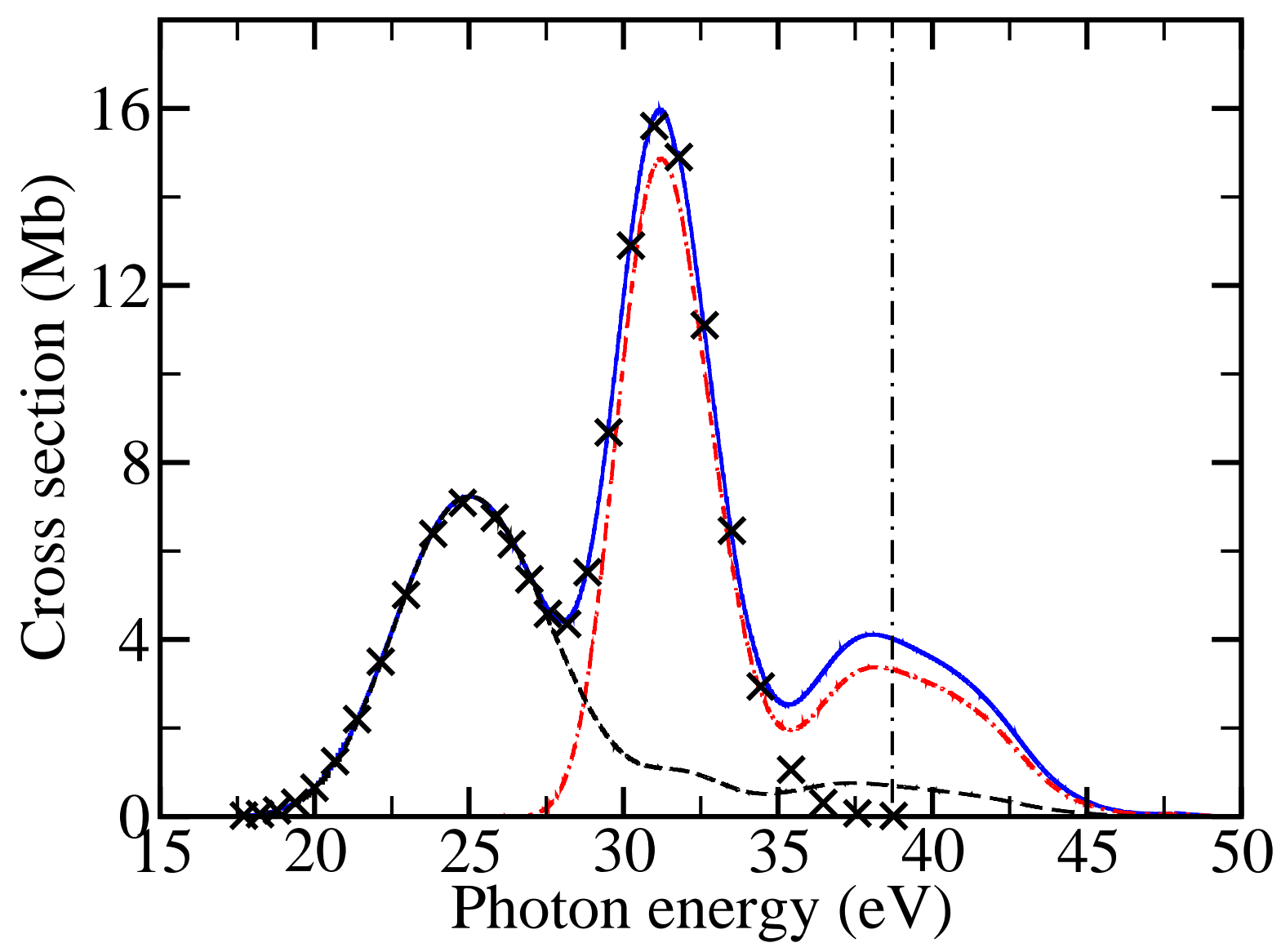

Figure 5. Total photodissociation cross section of $\mathrm{HeH}^{+}$after averaging over an isotropic orientational distribution (blue solid). Also shown are the partial contributions from parallel (black dashes) and perpendicular (red chain) orientations. The calculation is performed for transitions starting from the lowest lying vibrational state $\nu=0$ and from $J=1$. The crosses are the theoretical result from [7] and the vertical chain at $38.7 \mathrm{eV}$ marks the experimental photon energy.

parallel contribution, while above about $29 \mathrm{eV}$ the perpendicular contribution is clearly dominant. Similarly to the parallel case the perpendicular spectrum shows a dominant low-energy peak followed by a rather structureless tail. In fact, the tail looks more like a second peak, but it is composed of a number of electronic transitions. In comparison to the tail for a parallel alignment the one in the perpendicular case is confined to a smaller energy window.

An older calculation [7] (performed using the length gauge) took into account only three excited states of $\mathrm{HeH}^{+}$, namely the first two excited $\Sigma$ states and the first $\Pi$ state. Figure [5 shows that for these particular states the agreement between the present calculation and the one from [7] is excellent, but at the photon energy used in the FLASH experiment higher lying states clearly dominate the spectrum. 


\subsection{Comparison to experiment: channel separation, diabatic effects, and vibrational} excitation

The heteronuclear $\mathrm{HeH}^{+}$ion can dissociate into two different channels: $\mathrm{He}+\mathrm{H}^{+}$and $\mathrm{H}+\mathrm{He}^{+}$. The FLASH experiment [11] measured only the cross section given by the dissociation of $\mathrm{HeH}^{+}$into $\mathrm{He}+\mathrm{H}^{+}$. A comparison requires thus a separation of the two channels. Within the adiabatic approximation the asymptotic long-range behaviour, i. e. the separated atom limit at $R \rightarrow \infty$, defines the channel into which the population of a given adiabatic electronic state dissociates. At $R=60 a_{0}$ the potential curves were found to have almost reached the separated atom limit. A comparison of the asymptotic potential-curve energies with the sum of the energies of an independent atom and ion $\left(\mathrm{He}+\mathrm{H}^{+}\right.$or $\left.\mathrm{H}+\mathrm{He}^{+}\right)$in their various states yielded a well-defined channel assignment. The result is shown in figures 1 and 2. Most of the lower lying states dissociate into $\mathrm{He}+\mathrm{H}^{+}$while the highest lying Rydberg states dissociate into $\mathrm{H}+\mathrm{He}^{+}$. The two blocks are rather well separated, except for three states (for both ${ }^{1} \Sigma$ and ${ }^{1} \Pi$ symmetry) that dissociate into $\mathrm{H}+\mathrm{He}^{+}$but lie in the energy regime dominated by the $\mathrm{He}+\mathrm{H}^{+}$ channel.

After considering only transitions to electronic states that are assigned to the He $+\mathrm{H}^{+}$channel the partial photodissociation cross section shown in figure 6 is obtained. The result (obtained for $\nu=0$ and $J=1$ ) shows in comparison to figure 5 that overall dissociation into the $\mathrm{He}+\mathrm{H}^{+}$channel is clearly less favoured. However, the relative importance of the two channels varies strongly with photon energy. Below about $27 \mathrm{eV}$ the spectrum stems solely from the first excited state of ${ }^{1} \Sigma$ symmetry which dissociates into $\mathrm{H}+\mathrm{He}^{+}$. In between about 30 and $35 \mathrm{eV}$ the total dissociation cross section is dominated by the contribution from the lowest lying ${ }^{1} \Pi$ state that also dissociates into $\mathrm{H}+\mathrm{He}^{+}$. A smaller contribution comes, however, from the second excited ${ }^{1} \Sigma$ state. This state is responsible for the first peak observed in the $\mathrm{He}+\mathrm{H}^{+}$channel in figure 6. Thus the parallel contribution is equal to the total cross section of this channel in that energy range. Above about $35 \mathrm{eV}$ many other states contribute, and the cross sections for the two dissociation channels are almost of equal size, as a comparison of figures 5 and 6 shows. Since the majority of the states dissociating into $\mathrm{He}+\mathrm{H}^{+}$lies energetically above the ones dissociating into $\mathrm{H}+\mathrm{He}^{+}$, the maximum of the second peak of the $\mathrm{He}+\mathrm{H}^{+}$cross section is shifted to slightly higher energy than the one of the total dissociation cross section.

For comparison, figure 6] shows also the experimentally determined absolute partial dissociation cross section at $38.7 \mathrm{eV}$ for the $\mathrm{He}+\mathrm{H}^{+}$channel as well as its parallel contribution [11]. The agreement is almost perfect, but further aspects have to be considered for a direct comparison. First, the present analysis is based on an adiabatic model. Second, it appears to be clear that the $\mathrm{HeH}^{+}$ions investigated in the experiment were not in their vibrational ground state. These two issues are thus discussed in the remainder of this section.

The potential curves in figures 1 and 2 show a number of avoided crossings. Clearly, 


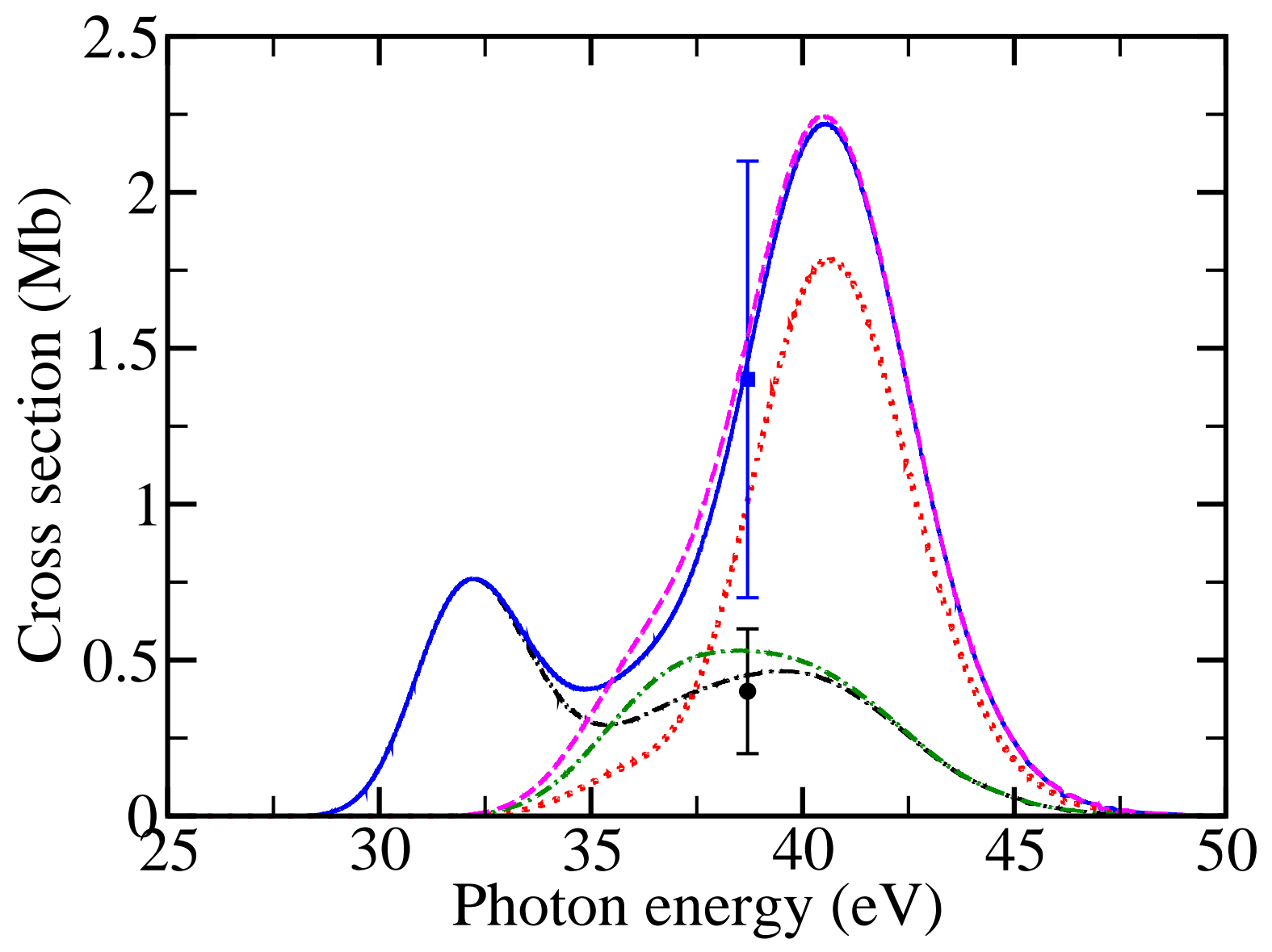

Figure 6. Partial photodissociation cross section of $\mathrm{HeH}^{+}$yielding $\mathrm{He}+\mathrm{H}^{+}$. The calculations are performed for transitions starting from the lowest lying vibrational state $\nu=0$ and from $J=1$. The blue square is the experimental total cross section and the black circle is the experimental parallel contribution (both from [11). Adiabatic limit: the blue solid line represents the isotropic orientation of the molecular axis with respect to the field. The parallel (black chain) and perpendicular (red dots) contributions are also shown. Diabatic limit: the magenta dashes represent the isotropic orientation, while the green chain is the parallel contribution.

at these crossings there is some probability for a transition from one adiabatic curve to the other. In the present context not all avoided crossings are, however, of equal importance. Those between states dissociating into the same dissociation channel do not change the partial cross section into, e.g., the $\mathrm{He}+\mathrm{H}^{+}$channel. (Clearly, these avoided crossing have to be considered in a calculation of other differential spectra like the kinetic-energy distribution of the ionic or atomic fragments.) A more careful analysis that takes also the relative importance of the different electronic states (at the experimental photon energy) into account shows that the most critical avoided crossing for the present discussion is the one occuring close to $R=4.0 a_{0}$ between the 2 nd and 3rd excited state of ${ }^{1} \Sigma$ symmetry (see figure 1). (This avoided crossing is already visible in the theoretical data in [2, 16] and thus reproduced by other numerical methods.)

A standard procedure for estimating the importance of diabatic effects at an avoided 
curve crossing is provided by the Landau-Zener approximation (see, e.g., Chapter 10 in [17] and references therein). Within this model the probability for a transition from one adiabatic curve to the other is given by

$$
P_{12}^{a}=\exp \left[-\frac{2 \pi H_{12}\left(R_{c}\right)^{2}}{\hbar v\left|F_{1}-F_{2}\right|}\right],
$$

where $R_{c}$ is the internuclear separation at which the avoided crossing occurs, $H_{12}\left(R_{c}\right)$ is the diabatic coupling which can be calculated from the relation $E_{2}-E_{1}=2 H_{12}\left(E_{1}\right.$ and $E_{2}$ being the adiabatic energies), the velocity $v$ is obtained from the kinetic energy and $F_{1,2}$ are the slopes of the diabatic curves at $R_{c}$. In the present case, it turns out that the Landau-Zener formula is difficult to apply, since the two curves cross in a region where their behaviour is not at all linear. (A removal of the nuclear repulsion term does not resolve this problem.) As a consequence, it is difficult to estimate the slopes and the result of the Landau-Zener estimate has such a large error bar that it is practically needless. Consequently, figure 6 shows the cross sections in the two extreme cases: the already discussed adiabatic limit $\left(P_{12}^{a}=0\right)$ and the fully diabatic one $\left(P_{12}^{a}=1\right)$. From the figure one may conclude that the influence of possible diabatic effects on the cross section at the photon energy used in the experiment is not very relevant. The most noticeable changes would take place at lower photon energies.

All the spectra shown so far were obtained by assuming that the system starts in the lowest lying vibrational state $\nu=0$. This is valid for thermal distributions at not too high temperatures. However, due to the way the $\mathrm{HeH}^{+}$ions are generated in the FLASH experiment reported in [11] the detailed vibrational distribution is unknown, but not expected to be thermal. In a more recent experiment on $\mathrm{He}_{2}^{+}$in which the ions were produced in the same way (by heavy particle collisions) the vibrational distribution was determined [18]. In this case the initial-state distribution was found to be distributed over at least 5 vibrational states $(\nu=0$ to 4$)$. Since the binding energy of $\mathrm{He}_{2}^{+}$is similar to that of $\mathrm{HeH}^{+}$, one may expect a similar vibrational-state distribution for both ions. Therefore, photodissociation cross sections for transitions starting from different vibrational states were computed. The ones corresponding to the initial states $\nu=0,1,2$, and 3 are shown in figure 7 .

At the experimental photon energy the results for both the adiabatic and diabatic limit are very close to each other, but there are evident differences at lower photon energies. Accidentally, the photon energy in [11] probes the spectrum of the $\mathrm{HeH}^{+}$ion at a point where the cross sections of the $\nu=0$ and $\nu=1$ states cross each other. As a consequence, the performed experiment is rather insensitive to a redistribution of population between the states with $\nu=0$ and $\nu=1$. The population of higher vibrational states $(\nu>1)$ leads on the other hand to a decrease of the cross section at $38.7 \mathrm{eV}$. The trend visible for $\nu=2$ and $\nu=3$ continues for higher values of $\nu$, as was checked though not explicitly shown. 
Photodissociation of the $\mathrm{HeH}^{+}$molecular ion
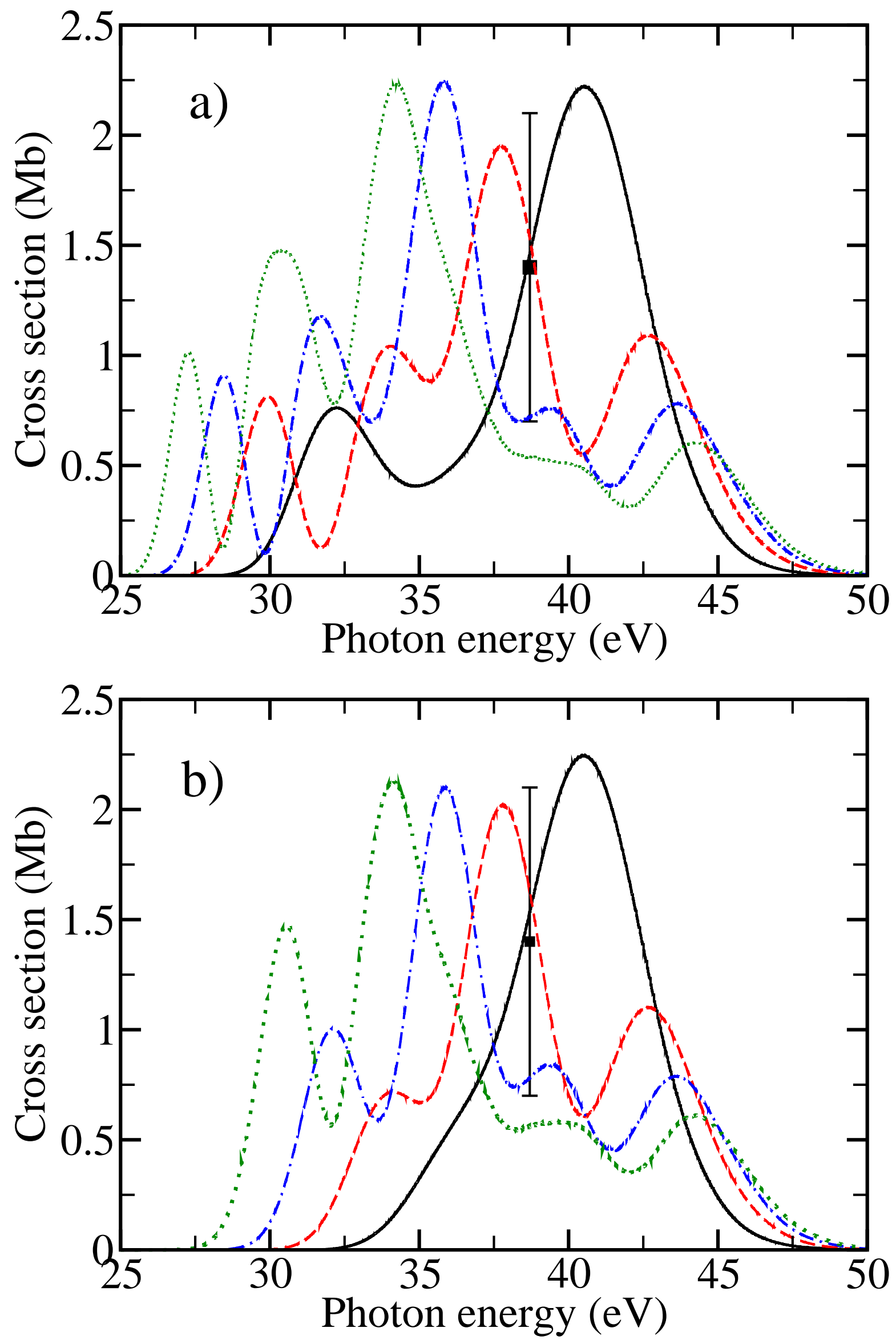

Figure 7. Partial photodissociation cross section of $\mathrm{HeH}^{+}$decaying into $\mathrm{He}+\mathrm{H}^{+}$ 


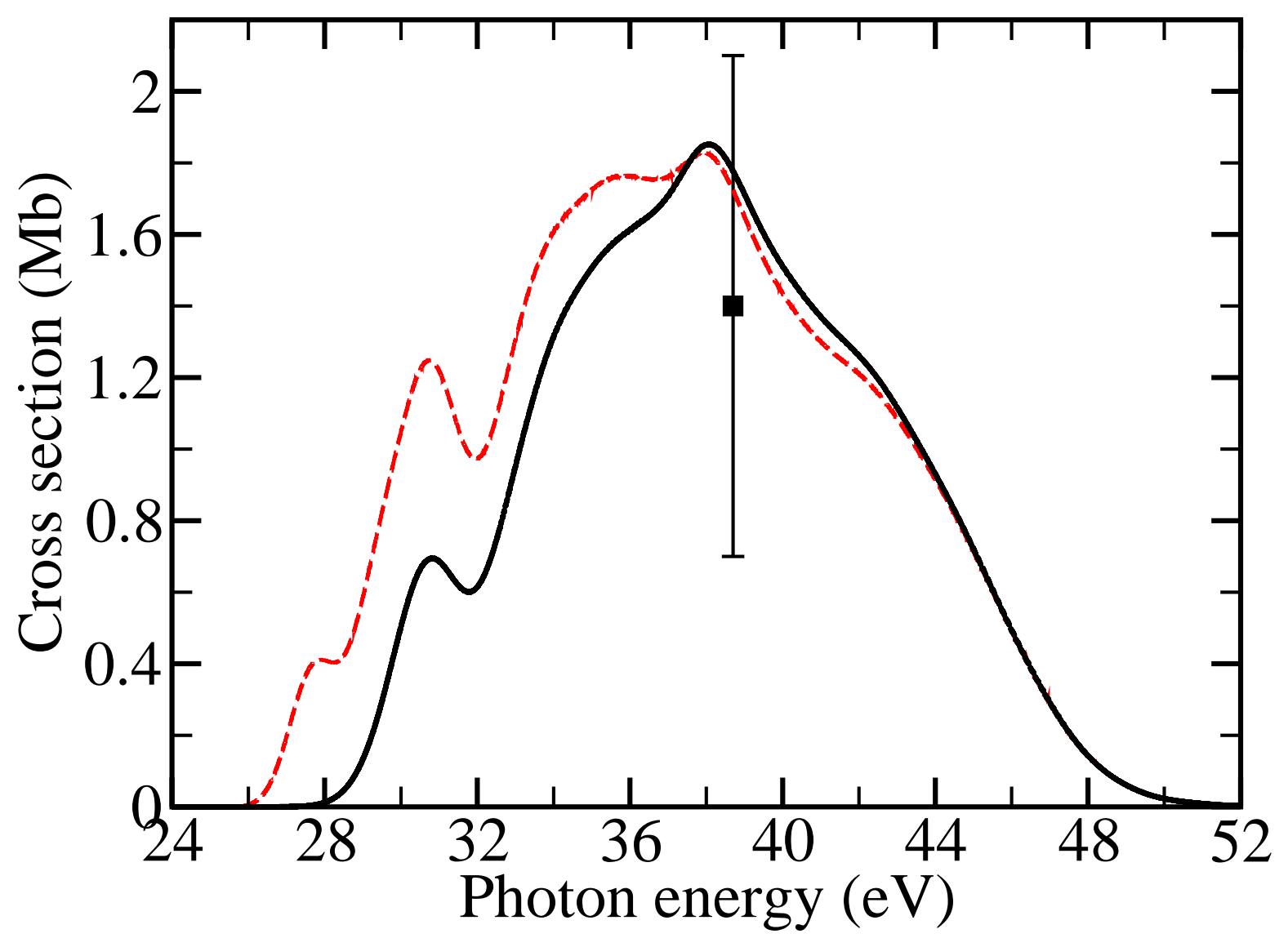

Figure 8. Photodissociation of $\mathrm{HeH}^{+}$into $\mathrm{He}+\mathrm{H}^{+}$- comparison between theoretical calculations (adiabatic - red dashes, and diabatic estimate - black solid line) for transitions starting from a mixture of vibrational levels $(33 \% \nu=0,45 \% \nu=1$, and $22 \% \nu \geq 2$ ) and initial rotational level $J=1$ with the experimental (black square) result [1].

If one assumes the initial vibrational distribution of $\mathrm{HeH}^{+}$in [1] to be identical to the one of $\mathrm{He}_{2}^{2+}$ in [18], the photodissociation cross section shown in figure 8 is obtained. The vibrational distribution is in this case modelled in the following way: $33 \%$ of the vibrational population was considered to be in the $\nu=0$ level, $45 \%$ in $\nu=1$, and $22 \%$ are equally distributed over the states $\nu=2,3,4$. Compared to figure 6 the influence of the vibrational distribution is obvious: the shape of the spectrum changes and the value of the cross section at the experimental photon energy $(38.7 \mathrm{eV})$ increases slightly (from $\sigma \approx 1.50 \mathrm{Mb}$ - see figure [6 - to $\sigma \approx 1.70 \mathrm{Mb}$ ). Thus the agreement to experiment decreases, but remains still well within the error bars of the measured cross section. In view of the lack of knowledge about the experimental initial vibrational distribution, it is, however, impossible to finally conclude on the degree of agreement between theory and experiment. As the present study shows, this distribution has a rather strong influence on the photodissociation cross section. In fact, the theoretical results may indicate a narrower vibrational distribution than the one found for $\mathrm{He}_{2}^{2+}$ in [18] that was assumed to be similar to the one of $\mathrm{HeH}^{+}$in [11]. It would certainly be of interest to have experimental data also for other photon energies. This may also help 
to (indirectly) determine the initial vibrational-state distribution. Furthermore, these data may be an interesting direct measure of the adiabaticity at the discussed avoided crossing between the 2 nd and $3 r d$ excited state of ${ }^{1} \Sigma$ symmetry.

\section{Summary}

In this work the photodissociation cross section of $\mathrm{HeH}^{+}$was computed. A previous theoretical result that considered a parallel alignment of the molecular axis was confirmed. A calculation for a perpendicular alignment was performed that considered a large number of electronic states in order to obtain a converged photodissociation cross section. In order to compare to a recent experiment, the partial cross sections for dissociation into either $\mathrm{He}+\mathrm{H}^{+}$or $\mathrm{H}+\mathrm{He}^{+}$were determined. This was done within the adiabatic approximation, but possible diabatic effects were estimated. Different initial vibrational and rotational levels were considered and photodissociation spectra for transitions starting from the rotational levels $J=0,1$ and vibrational levels $\nu=0,1,2,3,4$ were presented. The influence of the vibrational motion on the final spectra was investigated and compared to the experimental findings. Agreement within the experimental error bar was found, but it was concluded that the knowledge of the experimental vibrational distribution is important for a final conclusion. The present analysis should motivate further experimental studies, especially at variable photon energy, as it should become possible due to the current progress in the development of free-electron lasers.

\section{Acknowledgments}

The authors acknowledge helpful discussions with A. Wolf and financial support by the Deutsche Forschungsgemeinschaft through the SFB 450, the Stifterverband für die Deutsche Wissenschaft, the European COST Action CM0702, and the Fonds der Chemischen Industrie.

\section{References}

[1] H. H. Michels. Molecular orbital studies of the ground and low-lying excited states of the $\mathrm{HeH}^{+}$ molecular ion. J. Chem. Phys., 44:3834, 1966.

[2] T. A. Green, H. H. Michels, J. C. Browne, and M. M. Madsen. Configuration interaction studies of the $\mathrm{HeH}^{+}$molecular ion. I Singlet sigma states. J. Chem. Phys., 61:5186, 1974.

[3] T. A. Green, H. H. Michels, J. C. Browne, and M. M. Madsen. Configuration interaction studies of the $\mathrm{HeH}^{+}$molecular ion. II Dipole and radial coupling matrix elements for the singlet sigma states. J. Chem. Phys., 61:5198, 1974.

[4] T. A. Green, H. H. Michels, and J. C. Browne. Configuration interaction studies of the $\mathrm{HeH}^{+}$ molecular ion. III Singlet pi and delta states. J. Chem. Phys., 64:3951, 1976.

[5] T. A. Green, H. H. Michels, and J. C. Browne. Configuration interaction studies of the $\mathrm{HeH}^{+}$ molecular ion. IV The triplet sigma, pi, and delta states. J. Chem. Phys., 69:101, 1978.

[6] Samir Saha, K. K. Datta, and A. K. Barua. Photodissociation of $\mathrm{HeH}^{+}$by both electronic and vibrational transitions. J. Phys. B: At. Mol. Phys., 11:3349, 1978. 
[7] D Basu and A K Barua. Photodissociation of $\mathrm{HeH}^{+}$. J. Phys. B: At. Mol. Phys., 17:1537, 1984.

[8] A. Saenz. Photoabsorption and photoionization of $\mathrm{HeH}^{+}$. Phys. Rev. A, 67:033409, 2003.

[9] Michele Pavanello, Sergiy Bubin, Marcin Molski, and Ludwik Adamowicz. Non-Born-Oppenheimer calculations of the pure vibrational spectrum of $\mathrm{HeH}^{+}$. J. Chem. Phys., 123:104306, 2005.

[10] Bing-Lu Zhou, Jiong-Ming Zhu, and Zong-Chao Yan. Ground-state energy of $\mathrm{HeH}^{+}$. Phys. Rev. A, 73:064503, 2006.

[11] H. B. Pedersen, S. Altevogt, B. Jordon-Thaden, O. Heber, M. L. Rappaport, D. Schwalm, J. Ullrich, D. Zajfman, R. Treusch, N. Guerassimova, M. Martins, J.-T. Hoeft, M. Wellhófer, and A. Wolf. Crossed beam photodissociation imaging of $\mathrm{HeH}^{+}$with vacuum ultraviolet freeelectron laser pulses. Phys. Rev. Lett., 98:223202, 2007.

[12] J. Fernández and F. Martín. Photoionization of the $\mathrm{HeH}^{+}$molecular ion. J.Phys. B: At. Mol. Phys., 40:2471, 2007.

[13] Irina Dumitriu, Yulian V Vanne, Manohar Awasthi, and Alejandro Saenz. Photoionization of the alkali dimer cations $\mathrm{Li}_{2}^{+}, \mathrm{Na}_{2}^{+}$and $\mathrm{LiNa}^{+}$. J. Phys. B: At. Mol. Phys., 40:1821, 2007.

[14] Yulian V. Vanne and Alejandro Saenz. Numerical treatment of diatomic two-electron molecules using a B-spline based CI method. J. Phys. B: At. Mol. Phys., 37:4101, 2004.

[15] Yulian V. Vanne, Alejandro Saenz, Alex Dalgarno, Robert C. Forrey, Piotr Froelich, and Svante Jonsell. Doubly excited autoionizing states of $H_{2}$ converging to the $H(n=2)+H\left(n^{\prime}=2\right)$ limit. Phys. Rev. A, 73:062706, 2006.

[16] S. Jonsell, A. Saenz, and P. Froelich. Neutrino-mass determination from tritium $\beta$ decay: Corrections to and prospects of experimental verification of the final-state spectrum. Phys. Rev. C, 60:034601, 1999.

[17] Gert D. Billing and Kurt V. Mikkelsen. Molecular Dynamics and Chemical Kinetics. John Wiley and Sons, Inc., 1996.

[18] H. Buhr, H. B. Pedersen, S. Altevogt, V. M. Andrianarijaona, H. Kreckel, L. Lammich, S. Novotny, D. Strasser, J. Hoffmann, M. Lange, M. Lestinsky, M. B. Mendes, M. Motsch, O. Novotný, D. Schwalm, X. Urbain, D. Zajfman, and A. Wolf. Inelastic electron collisions of the isotopically symmetric helium dimer ion ${ }^{4} \mathrm{He}_{2}^{+}$in a storage ring. Phys. Rev. A, 77:032719, 2008. 